\title{
La depresión de los padres se asoció a un menor rendimiento escolar en adolescentes
}

Parental depression was associated with decreased child school performance

Shen H y col. JAMA Psychiatry. 2016;73(3):239-246.

\section{Objetivos}

Evaluar las asociaciones entre depresión materna y paterna con el rendimiento escolar de los niños al final de la educación obligatoria (aproximadamente a los 16 años).

\section{Diseño, lugar y pacientes}

Estudio de cohorte a escala nacional realizado en Suecia. Se incluyeron jóvenes nacidos en ese país entre 1984 y 1994 (n $=1.124 .162$ ), 93,9\% de las personas nacidas en Suecia durante ese período. Los criterios de exclusión fueron los niños adoptados, los que no podían estar vinculados a su madre o padre biológicos, y aquellos que no tenían datos sobre su rendimiento escolar. Se realizó además un análisis de una muestra de jóvenes que habían residido en el condado de Estocolmo durante el estudio para evaluar si una definición más amplia de la depresión parental influía en los resultados. Esta muestra incluyó 214.668 personas $(19,1 \%$ de la muestra nacional).

\section{Evaluación de los datos}

Los datos sobre el rendimiento escolar y los diagnósticos de depresión se recolectaron del registro nacional escolar y de los registros de los hospitales, respectivamente. En la submuestra, el diagnóstico de depresión se basó tanto en los datos del registro nacional como en los del registro regional de los centros ambulatorios, de especialistas y de tratamiento del condado de Estocolmo. Para realizar el análisis se tuvieron en cuenta covariables que habitualmente se asocian con el rendimiento escolar como: sexo, año de nacimiento y orden de nacimiento del niño; edad materna y paterna al nacimiento del niño; tabaquismo materno durante el embarazo; nivel de educación de los padres; ingreso familiar ajustado por año y tamaño de la familia; región parental de nacimiento y abuso de alcohol por parte de los padres antes del nacimiento del niño.

\section{Resultados}

Tanto los modelos crudos como los ajustados (aunque, en menor medida) mostraron que el antecedente de depresión de los padres en cualquier momento antes del último año de escolaridad obligatoria de sus hijos estaba asociada a un menor rendimiento escolar de los jóvenes. Los resultados principales se resumen en la tabla 1.

Tabla 1. Asociación entre depresión en los padres y el rendimiento escolar al final de la escolaridad obligatoria en una cohorte de jóvenes nacidos entre 1984 y 1994.

\begin{tabular}{|c|c|c|c|c|}
\hline & \multicolumn{4}{|c|}{ Rendimiento escolar (Diferencia de medias, IC 95\%) } \\
\hline Variables & Muestra Total & Modelo $1^{\mathrm{b}}$ & Modelo $2^{\mathrm{c}}$ & Modelo $3^{\mathrm{d}}$ \\
\hline \multicolumn{5}{|l|}{ Población ( $n=1.124 .162)$} \\
\hline Depresión materna antes 16 años del niño $(n=33.906)$ & $-0,80(-0,83 \mathrm{a}-0,77)$ & $-0,81(-0,84 \mathrm{a}-0,78)$ & $-0,61(-0,64 \mathrm{a}-0,58)$ & $-0,45(-0,48 \mathrm{a}-0,42)$ \\
\hline Depresión paterna antes de los 16 años niño $(n=23.724)$ & $-0,73(-0,77 \mathrm{a}-0,69)$ & $-0,72(-0,76 \mathrm{a}-0,69)$ & $-0,61(-0,64 \mathrm{a}-0,57)$ & $-0,40(-0,43 \mathrm{a}-0,37)$ \\
\hline \multicolumn{5}{|l|}{ Muestra del condado de Estocolmo $(n=214$ 668) } \\
\hline Depresión materna antes 16 años del niño $(n=16.138)$ & $-0,67(-0,72 \mathrm{a}-0,62)$ & $-0,75(-0,80 \mathrm{a}-0,71)$ & $-0,59(-0,63 \mathrm{a}-0,54)$ & $-0,44(-0,48 \mathrm{a}-0,39)$ \\
\hline Depresión paterna antes de los 16 años niño $(n=8.837)$ & $-0,47(-0,53 \mathrm{a}-0,40)$ & $-0,49(-0,55 a-0,43)$ & $-0,44(-0,50 \mathrm{a}-0,38)$ & $-0,27(-0,32 \mathrm{a}-0,21)$ \\
\hline
\end{tabular}

aEn una escala de 1 (peor) a 10 (mejor). bModelo 1 ajustado según las características del niño (sexo, nacimientos múltiples y Año de nacimiento). cModelo 2 ajustado para el modelo 1 y las características del embarazo (edad materna al nacer, orden de nacimiento y tabaquismo materno en la primera consulta prenatal). dModelo 3 ajustado para el modelo 2 y características familiares (edad paterna, educación de los padres, abuso de alcohol por parte de los padres antes del nacimiento del niño, quintil de ingreso familiar anual ajustado por año y tamaño de la familia, y parental región de nacimiento).

Además, se observó que la edad menor de los progenitores y el tabaquismo materno durante el embarazo tuvieron asociación con un menor rendimiento escolar y que, por el contrario, el mayor ingreso económico familiar, nivel de educación paterna y el origen de los padres de Suecia con un mejor rendimiento en los jóvenes. Luego del análisis de distintas covariables se evidenció que tamaño del efecto de la depresión de los padres durante múltiples períodos de desarrollo del niño se asoció de manera independiente con un menor rendimiento escolar. Además se demostró que, si bien en la muestra nacional, la depresión materna y paterna tuvieron asociaciones similares con el rendimien- to escolar, en la submuestra, la depresión materna tuvo un efecto adverso negativo mayor que la paterna. Por último, se expuso que la depresión materna tuvo una mayor influencia negativa en el rendimiento escolar de las mujeres que de los varones estudiados.

\section{Conclusiones}

Los resultados sugieren que el diagnóstico de depresión en los padres puede tener un efecto de gran alcance sobre el desarrollo de los hijos.

Conflicto de interés de los autores: no referido. 


\section{Comentario}

La depresión es una importante causa de morbilidad y discapacidad en todo el mundo ${ }^{1}$ no solo por lo que ocasiona en la persona deprimida sino por sus repercusiones en el contexto, en especial en los niños del núcleo familiar. Si bien varios estudios mostraron ${ }^{2,3}$ que existe una asociación entre la depresión de los padres y los problemas en el desarrollo neurológico, conductual, psíquico y emocional de sus hijos, hasta el momento solo unos pocos estudios han asociado la depresión de los padres con el bajo rendimiento escolar. La importancia en el desempeño escolar radica en que es un poderoso predictor de salud y que, junto con la ocupación y los ingresos, condiciona la posición social de un individuo, otro de los predictores más fuertes de salud y mortalidad futuras. En este estudio de más de 1 millón de jóvenes de una cohorte nacional en Suecia, la depresión materna y la paterna se asociaron independientemente con menor rendimiento académico a los 16 años de edad. El ajuste para una amplia gama de covariables atenuó las asociaciones, lo que sugiere que existen factores de confusión, aunque se mantuvo la evidencia de un efecto de la depresión parental. Si bien se puede pensar que existe una responsabilidad genética, ya que la depresión prenatal también se asoció a un bajo rendimiento escolar, no se puede soslayar el deterioro que se genera en el vínculo entre padres e hijos cuando un progenitor sufre depresión. El hecho de que en la submuestra la depresión materna superara a la paterna en los efectos adversos y que tuvo una mayor influencia en el rendimiento escolar de las mujeres en comparación con los varones, es consistente con los resultados de estudios previos $4,5,6$ que sugieren que las mujeres pueden ser más sensibles a los efectos adversos de la depresión materna. Una explicación es que las relaciones de madre-hija y padre-hija son diferentes. Entre las fortalezas del estudio se pueden destacar el largo seguimiento, el gran tamaño de muestra, el ajuste para muchos posibles factores de confusión y la evaluación objetiva de la depresión. Otra ventaja es que los datos provienen de Suecia, y hasta ahora, los estudios de descendientes de padres deprimidos han sido de los Estados Unidos, Holanda, Reino Unido, Alemania y Australia, lo que demuestra la universalidad de los hallazgos, al menos en los países occidentales. La principal limitación del estudio fue el subdiagnóstico de la depresión dado que los registros estaban basados en el uso de un servicio público que pudo subestimar el verdadero grado de depresión en la población; sin embargo, en el análisis de la submuestra, con mejor comprobación, también se demostraron asociaciones similares entre la depresión parental y el rendimiento escolar.

\section{Conclusiones de la comentadora}

Los hallazgos muestran claramente que tanto la depresión materna como paterna que ocurre en cualquier momento de la vida de un niño hasta los 16 años de edad se asocia con un ligero deterioro en el desempeño escolar del niño. Debido a que la depresión de los padres puede ser un factor de riesgo modificable y más susceptible de mejora en comparación con otros (como la situación socioeconómica), vale la pena poner esfuerzo en diagnosticar y tratar en forma efectiva (fármacos y psicoterapia) la depresión de los adultos para ayudar a sus hijos.

Mercedes Mutchinick [ Servicio de Medicina Familiar y Comunitaria, Hospital Italiano de Buenos Aires. mercedes.mutchinick@ hospitalitaliano.org.ar ]

Mutchinick M. La depresión de los padres se asoció a un menor rendimiento escolar en adolescentes. Evid Actual Pract Ambul 2018;21(3):86-87. Comentado de: Shen $\mathrm{H}$ y col. Associations of Parental Depression With Child School Performance at Age 16 Years in Sweden. JAMA Psychiatry. 2016;73(3):239-246. PMID: 26842307

\section{Referencias}

1. Murray CJ, y col. Disability-adjusted life years (DALYs) for 291diseases and injuries in 21 regions, 1990-2010: a systematic analysis for the Global Burden of Disease Study 2010. Lancet. 2012;380(9859):2197-2223.

2. Mackrell SV, y col. Child temperament and parental depression predict cortisol reactivity to stress in middle childhood. J Abnorm Psychol. 2014;123(1):106-116. 3. Luoma I, y col. Longitudinal study of maternal depressive symptoms and child well-being.J Am Acad Child Adolesc Psychiatry. 2001;40(12):1367-1374.

4. Bouma EM, y col. Stressful life events and depressive problems in early adolescent boys and girls: the influence of parental depression, temperament and family environment.J Affect Disord. 2008;105(1-3):185-193.

5. Cummings EM, y col. Prospective relations between parental depression, negative expressiveness, emotional insecurity, and children's internalizing symptoms. Child Psychiatry Hum Dev. 2013;44(6):698-708.

6. Goodman SH, y col. Maternal depression and child psychopathology: a meta-analytic review. Clin Child Fam Psychol Rev. 2011;14(1):1-27.

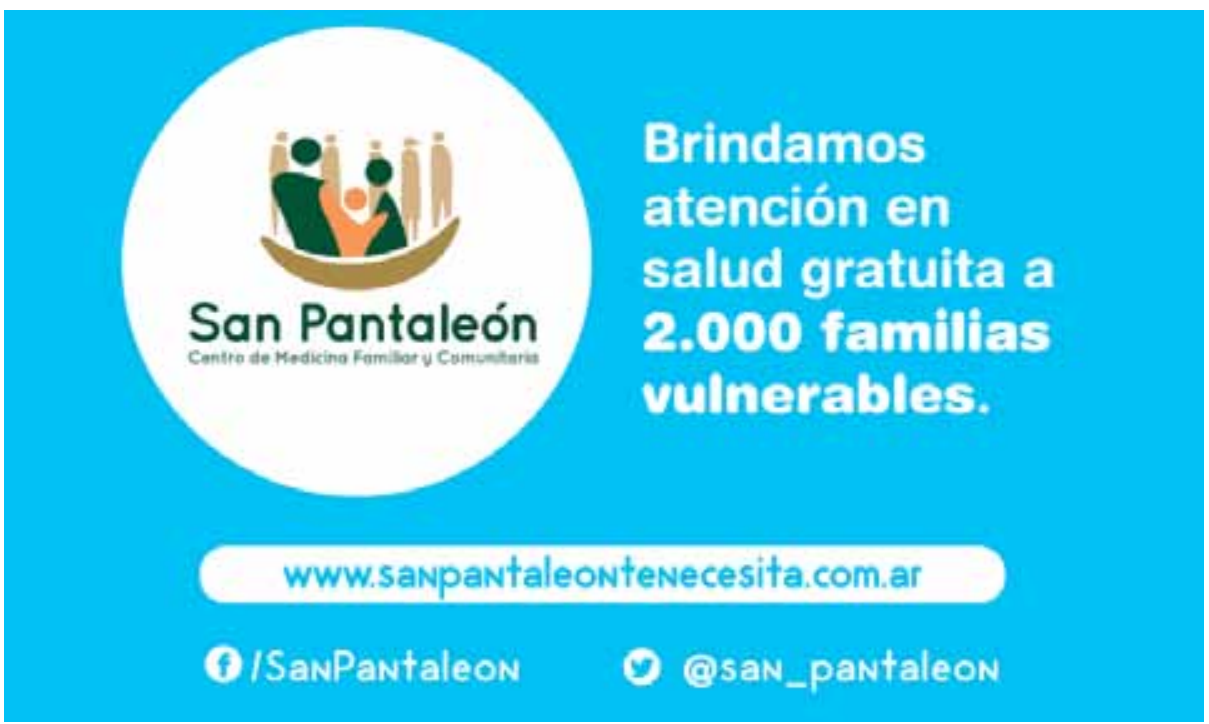

